\title{
Prevalence of intestinal parasites and related risk factors in rural localities from Pampa del Indio, Chaco, Argentina
}

\author{
Lindsay Renee Richards ${ }^{1}$, Cintia Delgado ${ }^{2}$, Marcia Goy ${ }^{3}$, Song Liang ${ }^{1}$, Maria \\ V. Periago ${ }^{2}$ \\ ${ }^{1}$ University of Florida, ${ }^{2}$ Fundación Mundo Sano, Argentina, ${ }^{3}$ Hospital Dr. Dante Tardelli, Argentina
}

Faculty mentor: Song Liang, Department of Environmental and Global Health

\begin{abstract}
Intestinal parasites are a significant cause of morbidity in endemic areas in many low- and middle-income countries (LMIC). Infections with intestinal parasites have been reported in multiple locations throughout Argentina, but infection prevalence is still unknown in many areas. The aim of this study was to determine the prevalence of intestinal parasites in rural areas surrounding Pampa del Indio, Chaco, Argentina, and to identify risk factors for human infections. In the current study, a survey of three rural neighborhoods surrounding the town of Pampa del Indio was conducted in July 2018. A total of 24 households were surveyed. A questionnaire to assess socio-economic and household variables was administered and fecal samples were collected. Of the 62 stool samples analyzed, an intestinal parasite prevalence of $46.8 \%$ ( 29 cases) was found. The most common parasite identified was Endolimax nana (22.6\%), followed by Giardia intestinalis (17.7\%), and Entamoeba coli (16.1\%). Most of the intestinal parasites found were protozoa, but three cases of helminths $(4.8 \%)$ were also identified. Participants were polyparasitized at a rate of $19.4 \%$. This study did not identify any statistically significant risk factors for infection but revealed a high overall rate of parasitism in the selected communities.
\end{abstract}

Keywords: intestinal parasites, protozoa, helminth, Argentina

\section{Background}

Intestinal parasites are a significant cause of morbidity in endemic areas in many low- and middle-income countries (LMIC). This includes infections with intestinal helminths, such as Ascaris lumbricoides, Trichiuris trichiuria, and hookworm, as well as infections with intestinal protozoans, such as Giardia intestinalis, Entamoeba histolytica, and Cryptosporidium spp. (Haque 2007). These infections can limit the health and nutritional status of hosts due to iron deficiency anemia, growth retardation, and various other mental and physical health issues (Liese et al. 2010). It is estimated that enteric protozoa are responsible for 2.9 million disabilityadjusted life years (DALY), while soil-transmitted helminths (STH) are responsible for another 3.9 million DALY (Daumerie \& Savioli 2010; Torgerson et al. 2015). The parasites can affect humans living in both rural and urban areas, and their infection prevalence has been linked with 
limited access to water, sanitation, and hygiene (WASH) factors (Hailegebriel 2017; Sah et al. 2013). Prior studies have shown that improvements to water supply can substantially reduce the rates of morbidity and severity of many parasitic infections (Esrey et al. 1991).

Infections with intestinal parasites have been reported in multiple locations throughout Argentina, but infection prevalence is still unknown in many areas (Dib et al. 2012; Gamboa et al. 2014; Zonta et al. 2010). A comprehensive understanding of the distribution of these parasites can inform public health policy and improve treatment efforts. The communities surrounding Pampa del Indio, Chaco, Argentina, were selected for this study due to their proximity and similarity to other areas known to have high rates of parasitism (Periago et al. 2018). Given the current lack of knowledge regarding parasite prevalence in these communities, this research investigates whether parasites can also be found in the selected area. The aim of this study is to determine the prevalence of intestinal parasites in these communities and to identify risk factors for their transmission.

\section{Methods}

\section{Study Area and Study Population}

A cross-sectional survey of three rural neighborhoods surrounding the city of Pampa del Indio, Argentina, was conducted in July 2018. Pampa del Indio is a municipality located within the Department of Libertador General San Martín in the Chaco province of northern Argentina, as shown in Figure 1. This department has a population of around 59,000 and a low population density of 7.6 inhabitants $/ \mathrm{km}^{2}$ (INDEC, 2010). Pampa del Indio is located within the sub-humid region of the Grand Chaco. The average rainfall for this region is between 750 to $1300 \mathrm{~mm}$ annually, and the temperature averages $21.8^{\circ} \mathrm{C}$ (Maldonado 2006; Espinosa et al. 2018).

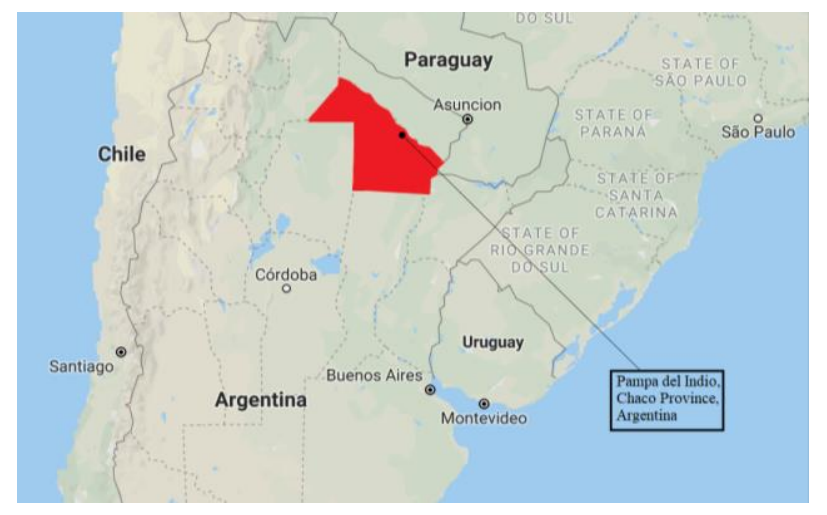

Figure 1. Map showing study area in Pampa del Indio, Chaco Province, Argentina (Google). 


\section{Study Design}

Three neighborhoods in the rural areas surrounding Pampa del Indio were surveyed: Fortín Brown, Tres Lagunas, and La Herradura. The research team visited all households in these neighborhoods to explain the study and determine whether household members wanted to participate. The households were georeferenced and consent forms were signed by all participating individuals, as well as by the guardians of any individuals under the age of 14 . A questionnaire was administered to collect data on all household members and to assess socioeconomic variables, such as education level, income, and main economic activity, in addition to household characteristics.

Households were defined as having unimproved roofs and walls if they were made from material other than bricks and cement and unimproved floors if they were dirt floors, illustrated in Figure 2. Bathrooms were considered unimproved if they lacked a latrine and septic tank, and water was considered unimproved unless it came from a water supply system or from bottled water. Because households typically did not have multiple bedrooms, those with five inhabitants or more were considered overcrowded.

\section{Stool Examination}

Next, single individual fecal samples were collected from consenting individuals. Stool containers labeled with household ID and individual names were distributed and collection was arranged for 2-3 days following the distribution. Collected samples were then transported to the Hospital Dante Tardelli (located in Pampa del Indio) for analysis.

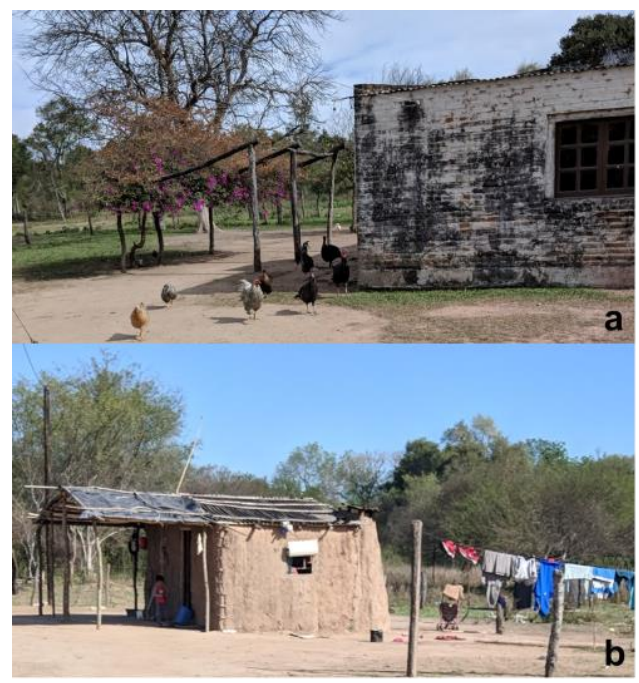

Figure 2. Households with (a) brick walls and (b) adobe walls in the neighborhoods surrounding Pampa del Indio, Chaco, Argentina. July 11, 2018. 
Samples were analyzed using three coprological techniques to identify parasites: Telemann sedimentation, Sheather flotation, and Willis flotation. Multiple techniques including both sedimentation and flotation were used in order to accurately assess the presence of parasites in each sample. Sedimentation is more appropriate for identifying eggs and cysts, while flotation is better suited for the identification of coccidian parasites (Periago et al. 2018). After samples were visualized under the microscope, images were compared against a diagnostic guide to identify parasites (Melvin, Brooke, \& Sadun 1965).

Telemann sedimentation. Between $5-10 \mathrm{~g}$ of each fecal sample was removed and mixed with a $5 \%$ formalin saline solution. After the mixtures were thoroughly homogenized, each was filtered through gauze into a $15 \mathrm{ml}$ centrifuge tube. Approximately $2 \mathrm{ml}$ of ether was added to each sample, and the tubes were then centrifuged for 5 minutes at 1000-15000 rpm. Supernatant was removed and the remaining sediment was observed under a microscope at 10x and 40x magnification (Telemann 1908).

Sheather flotation. A small amount of the concentrated fecal sample prepared via the Telemman technique was placed in an Eppendorf tube, which was then filled with an equal part of sugary solution. The tube was inverted several times to homogenize the mixture and then left to set for 5 minutes. Afterwards, a small amount of the solution was placed onto a slide and a coverslip was added. Slides were examined under 40x magnification (Sheather 1923).

Willis flotation. A small amount of the feces to be examined was placed in a container and the container was gradually filled to the brim with a salt solution. The container was left to set for 20 minutes to allow eggs to float to the top of the solution, at which point a glass slide was placed carefully on top of the container and then flipped over. A coverslip was added and the slide was moved to the microscope, where it was examined under 10x magnification (Willis 1921).

\section{Statistical Analysis}

Data was entered into Microsoft Excel (2010 Microsoft Corportation) and analyzed using R Version 3.5.2 (2018). Descriptive statistics of population means and standard deviations were calculated with 95\% confidence intervals (CI). A Fischer's Exact Test was used to calculate odds ratios and compare proportions of parasite infection with a level of statistical significance of $\mathrm{P}<$ 0.05 . 


\section{Results}

Descriptive characteristics of the study population and prevalence of intestinal parasites are shown in Table 1. A total of 24 households participated in this study. Containers were distributed to 117 individuals, and 62 fecal samples were collected (participation rate $=53.0 \%$ ). Participants ranged in age from < 1 year to 93 years old and $56.5 \%$ were females. Of the 62 stool samples analyzed, a prevalence of $46.8 \%$ ( 29 cases) was found. The most common parasite identified was Endolimax nana (22.6\%), followed by Giardia intestinalis (17.7\%), and Entamoeba coli $(16.1 \%)$. Most of the intestinal parasites found were protozoa, but three cases of helminths were also identified. A total of 12 individuals (19.4\%) were polyparasitized. The most common coinfections were Giardia intestinalis + Endolimax nana $(\mathrm{n}=3)$ and Endolimax nana + Chilomastix mesnili $(\mathrm{n}=2)$. No soil-transmitted helminths $(\mathrm{STH})$ were identified. Children under 14 and females had higher overall percentages of infections with intestinal parasites, but these differences were not statistically significant (Table 2).

\begin{tabular}{lc}
\hline Table 1. Descriptive Characteristics of Participants and Prevalence of Intestinal Parasites \\
\hline & Population Total \\
\hline Age (years), mean \pm SD & $33.8 \pm 25.4$ \\
$\quad$ Range & $0.75-93$ \\
Gender, $n(\%)$ & $35(56.5)$ \\
Female & $27(43.5)$ \\
Male & \\
Education, $n(\%)$ & $23(38.3)$ \\
Finished elementary school & $15(24.2)$ \\
Currently in school & $26[(41.9), 29.7-55.1]$ \\
Prevalence of protozoans, $n[(\%), 95 \% \mathrm{CI}]$ & $10[(16.1), 8.4-28.1]$ \\
Entamoeba coli & $1[(1.6), 0.08-9.8]$ \\
Iodamoeba butschlii & $5[(8.1), 3.0-18.5]$ \\
Chilomastix mesnili & $11[(17.7), 9.6-29.9]$ \\
Giardia intestinalis & $14[(22.6), 13.3-35.3]$ \\
Endolimax nana & $3[(4.8), 1.3-14.4]$ \\
Prevalence of helminths, $n[(\%), 95 \% \mathrm{CI}]$ & $1[(1.6), 0.08-9.8]$ \\
Enterobius vermicularis & $1[(3.2), 0.56-12.2]$ \\
Hymenolepis nana & $29[(46.8), 0.34-0.60]$ \\
Total intestinal parasite prevalence, $n[(\%), 95 \%$ CI $]$ &
\end{tabular}


Table 2. Characteristics of Participants Classified by Presence of Absence of Intestinal Parasites

\begin{tabular}{lccccc}
\hline Characteristics & $\begin{array}{c}\text { No. (\%) of Positive } \\
\text { Participants } \\
(\mathrm{n}=29)\end{array}$ & $\begin{array}{c}\text { No. (\%) of Negative } \\
\text { Participants } \\
(\mathrm{n}=33)\end{array}$ & OR (95\% CI) & P-value & $\begin{array}{c}\text { Total no. (\%) of } \\
\text { Participants (n = 62) }\end{array}$ \\
\hline Age Classification & & & & \\
$\quad$ Children* (0-13) & $13(44.8)$ & $9(27.3)$ & $\begin{array}{c}2.139(0.664- \\
7.184)\end{array}$ & 0.1881 & $22(35.5)$ \\
$\quad$ Adults (14+) & $16(55.2)$ & $24(72.7)$ & & & $40(64.5)$ \\
Gender & $17(58.6)$ & $18(54.5)$ & $1.177(0.384-$ & 0.8013 & $35(56.5)$ \\
$\quad$ Female* & $12(41.4)$ & $15(45.5)$ & $3.649)$ & $27(43.5)$ \\
$\quad$ Male & & & & & \\
\hline
\end{tabular}

Household characteristics as assessed in the surveys are shown below in Table 3. The most common source of primary income was animal farming, followed by pension or retirement. The mean number of inhabitants per household was 3.75, and 10 households (41.7\%) were classified as overcrowded. Most households had improved roofs, walls, and floors, and all households except for one had access to an electricity network. Effects of unimproved water could not be assessed because none of the households had improved water. Effects of unimproved bathrooms could also not be measured because all of the households had access to improved bathrooms. In accordance with the large quantity of individuals whose primary income came from farming, a high number of households had animal pens $(13 ; 54.2 \%)$. 
Table 3. Household Characteristics of Participants

Characteristics

Population Total

Primary income/livelihood, $n(\%)$

Mean no. of inhabitants per household

Houses with 5 inhabitants or more, $n(\%)$

Unimproved roof, $n(\%)$

Unimproved walls, $n(\%)$

Unimproved floor, $n(\%)$

Main cooking source, $n(\%)$

Main energy source, $n(\%)$

Main drinking water source, $n(\%)$

Main cooking water source, $n(\%)$

Main bathing/handwashing water source, $n(\%)$

Diaper disporal, $n(\%)$

Presence of kitchen garden, $n(\%)$

Presence of animal pen, $n(\%)$
19 (79.2) animal farm; 10 (41.7) pension/retirement; 3 (12.5) day laborer; 3 (12.5) social plan; 1 (4.2) field worker

$$
3.75
$$

$10(41.7)$

$3(12.5)$

$6(25)$

4 (16.7) gas; 15 (62.5) firewood; 5 (20.8) both

23 (95.8) electricity network; 1 (4.2) none

17 (70.8) borehole; 3 (12.5) school well; 2 (8.3) water truck; 1 (4.2) pump; 1 (4.2) mill

17 (70.8) borehole; 4 (16.7) school well; 1 (4.2) water truck; 1 (4.2) pump; 1 (4.2) mill

17 (70.8) borehole; 2 (8.3) school well; 2 (8.3) water truck; 1 (4.2) pump; 1 (4.2) mill; 1 (4.2) rain

18 (75) not relevant; 3 (12.5) toilet/latrine; 3 (12.5) trash

$8(33.3)$

$13(54.2)$

Odds ratios (OR) for different household characteristics were calculated using a Fisher's Exact Test and are shown in Table 4 below. Heterogeneity in infection prevalence was observed across different potential risk factors, but no factors showed significant associations with infection rate. 
Table 4. Household/Livelihood Characteristics and Their Association with the Presence/Absence of Intestinal Parasites

\begin{tabular}{|c|c|c|c|c|}
\hline $\begin{array}{l}\text { Characteristics } \\
\text { (risk factor*) }\end{array}$ & Presence of IP $(n=28)$ & Absence of IP $(n=32)$ & OR $(95 \% \mathrm{CI})$ & $P$-value \\
\hline \multicolumn{5}{|l|}{ Animal Farming } \\
\hline Yes* & $19(67.9)$ & $28(87.5)$ & \multirow{2}{*}{$0.308(0.060-1.301)$} & \multirow{2}{*}{0.1146} \\
\hline No & $9(32.1)$ & $4(12.5)$ & & \\
\hline \multicolumn{5}{|l|}{ Informal Slaughter } \\
\hline Yes* & $26(92.9)$ & $29(90.6)$ & \multirow{2}{*}{$1.338(0.142-17.195)$} & \multirow{2}{*}{1} \\
\hline No & $2(7.1)$ & $3(9.4)$ & & \\
\hline \multicolumn{5}{|l|}{ Kitchen Garden } \\
\hline Yes* & $13(46.4)$ & $10(31.3)$ & \multirow{2}{*}{$1.886(0.587-6.250)$} & \multirow{2}{*}{0.2906} \\
\hline No & $15(53.6)$ & $22(68.7)$ & & \\
\hline \multicolumn{5}{|l|}{ Roof } \\
\hline Unimproved* & $4(14.3)$ & $4(12.5)$ & \multirow{2}{*}{1.164 (0.194-6.974) } & \multirow{2}{*}{1} \\
\hline Improved & $24(85.7)$ & $28(87.5)$ & & \\
\hline \multicolumn{5}{|l|}{ Walls } \\
\hline Unimproved $*$ & $7(26.9)$ & $8(27.6)$ & \multirow{2}{*}{$0.968(0.346-3.733)$} & \multirow{2}{*}{1} \\
\hline Improved & $19(73.1)$ & $21(72.4)$ & & \\
\hline \multicolumn{5}{|l|}{ Floor } \\
\hline Unimproved* & $7(25.0)$ & $7(21.9)$ & \multirow{2}{*}{$1.187(0.301-4.695)$} & \multirow[b]{2}{*}{1} \\
\hline Improved & $21(75.0)$ & $25(78.1)$ & & \\
\hline \multicolumn{5}{|c|}{ Overcrowding $(n=62)$} \\
\hline Yes* & $20(69.0)$ & $20(60.6)$ & \multirow{2}{*}{$1.436(0.448-4.762)$} & \multirow{2}{*}{0.5978} \\
\hline No & $9(31.0)$ & $13(39.4)$ & & \\
\hline
\end{tabular}

\section{Discussion}

The overall prevalence of intestinal parasites found in this study was $46.8 \%$, which included both protozoa and helminths. Many of the parasites identified here overlap with those found in a study that took place in the peri-urban areas of Resistencia, another city the Chaco province of Argentina (Matzkin et al. 2000). Some of the parasites identified are transmitted via contaminated water (Giardia intestinalis, Entamoeba coli), while others are spread through direct contact (Enterobius vermicularis, Hymenolepis nana). No STH were identified in this study, despite their presence in other localities of northern Argentina (Krolewiecki et al. 2010). This could be because Pampa del Indio has a drier climate that is less conducive to parasite transmission relative to other endemic areas, or because individuals tended to live relatively far apart, thus limiting opportunities for the spread of infections between households. No risk factors showed statistical significance in predicting infection. 
The scope of this study is limited because it employed convenience, rather than random, sampling. Due to resource constraints, all households within a close proximity were tested rather than random samples of households from a broader area. The number of parasites detected may be lower than the true population prevalence because only one stool sample per individual was tested, but infected individuals may not pass eggs each day (Marti \& Koella 1993). Additionally, the small sample size ( $n=62$ for individuals; $n=24$ for households) inhibits what can be inferred from statistical analysis and led to the use of OR rather than other measures that require a larger sample size to accurately test for significance. This may have contributed to the lack of power regarding risk factors. However, the data offers information about the presence and prevalence of certain intestinal parasites that can contribute to a growing knowledge regarding the global distribution of these infections.

The infections identified in this study disproportionately afflict communities facing poverty (Haque 2007). In order to reduce global health disparities, the needs of vulnerable communities with limited access to clean water and proper waste disposal must be investigated and addressed. Beyond the suffering caused by individual morbidities, infections with intestinal parasites are responsible for many DALY worldwide and can also slow the economic development of communities by limiting abilities to work and learn (Drake et al. 2000). Future studies should continue to fill gaps in knowledge by investigating the intestinal parasite prevalence in previously unstudied areas.

\section{Conclusion}

This study revealed a high overall prevalence of intestinal parasites in the communities surrounding Pampa del Indio. Based on the limited scope of this data, no risk factors were identified for parasite transmission. Additional studies of greater breadth are urgently needed to further understand the parasite distribution in northern Argentina, investigate the links between social and environmental factors and intestinal parasite infection, and ultimately reduce the morbidity and associated socioeconomic impacts caused by these infections.

\section{Acknowledgements}

This project would not have been possible without tremendous effort from the employees of Fundación Mundo Sano or the financial support from the University of Florida International Center. We also extend our gratitude to Nicholas Diaz for his statistical advice and mentorship. 


\section{References}

Daumerie, D., \& Savioli, L. (2010). Working to overcome the global impact of neglected tropical diseases: first WHO report on neglected tropical diseases (Vol. 1). World Health Organization.

Dib, J., Oquilla, J., Lazarte, S. G., \& Gonzalez, S. N. (2012). Parasitic prevalence in a suburban school of Famaillá, Tucumán, Argentina. ISRN microbiology, 2012.

Drake, L. J., Jukes, M. C. H., Sternberg, R. J., \& Bundy, D. A. P. (2000, October). Geohelminth infections (ascariasis, trichuriasis, and hookworm): cognitive and developmental impacts. In Seminars in Pediatric Infectious Diseases (Vol. 11, No. 4, pp. 245-251). WB Saunders.

Espinosa, M., Di Fino, E. M. A., Abril, M., Lanfri, M., Periago, M. V., \& Scavuzzo, C. M. (2018). Operational satellite-based temporal modelling of Aedes population in Argentina. Geospatial health, 13(2).

Esrey, S. A., Potash, J. B., Roberts, L., \& Shiff, C. (1991). Effects of improved water supply and sanitation on ascariasis, diarrhoea, dracunculiasis, hookworm infection, schistosomiasis, and trachoma. Bulletin of the World Health Organization, 69(5), 609.

Gamboa, M. I., Giambelluca, L. A., \& Navone, G. T. (2014). Distribución espacial de las parasitosis intestinales en la ciudad de La Plata, Argentina.

Google (n.d.). [Pampa del Indio, Chaco, Argentina]. Retrieved January 8, 2019, from https://www.google.com/maps?safe=active \&q=pampa+del+indio\&um=1\&ie=UTF$8 \& s a=X \& v e d=0 a h U K E w j c h K a N v 7 n g A h U J e a w K H Y n S C z s Q \_A U I D i g B$

Hailegebriel, T. (2017). Prevalence of intestinal parasitic infections and associated risk factors among students at Dona Berber primary school, Bahir Dar, Ethiopia. BMC infectious diseases, 17(1), 362.

Haque, R. (2007). Human intestinal parasites. Journal of health, population, and nutrition, 25(4), 387.

INDEC. Censo Nacional de Población, Hogares y Viviendas 2010.

Krolewiecki, A. J., Ramanathan, R., Fink, V., McAuliffe, I., Cajal, S. P., Won, K., \& Lee, R. (2010). Improved diagnosis of Strongyloides stercoralis using recombinant antigen-based serologies in a community-wide study in northern Argentina. Clin. Vaccine Immunol., 17(10), 1624-1630.

Liese, B., Rosenberg, M., \& Schratz, A. (2010). Programmes, partnerships, and governance for elimination and control of neglected tropical diseases. The Lancet, 375(9708), 67-76.

Maldonado P. Atlas del Gran Chaco Americano. 1st ed. Buenos Aires: Agencia Alemana de Cooperación Técnica; 2006.

Marti, H., \& Koella, J. C. (1993). Multiple stool examinations for ova and parasites and rate of falsenegative results. Journal of clinical microbiology, 31(11), 3044-3045.

Matzkin, R., Galván, M., Miranda, O., Merino, D., \& Balbachán, S. (2000). Parasitosis entéricas en una población escolar periurbana de Resistencia, Chaco. Comun. Cient. Tec, 40, 197-200.

Melvin, D. M., Brooke, M. M., \& Sadun, E. H. (1965). Common intestinal helminths of man; life cycle charts/prepared by Dorothy M. Melvin, MM Brooke, and EH Sadun. 
Periago, M. V., García, R., Astudillo, O. G., Cabrera, M., \& Abril, M. C. (2018). Prevalence of intestinal parasites and the absence of soil-transmitted helminths in Añatuya, Santiago del Estero, Argentina. Parasites \& vectors, 11(1), 638.

Sah, R. B., Bhattarai, S., Yadav, S., Baral, R., Jha, N., \& Pokharel, P. K. (2013). A study of prevalence of intestinal parasites and associated risk factors among the school children of Itahari, Eastern Region of Nepal. Tropical parasitology, 3(2), 140.

Sheather, A. L. (1923). The Detection of Intestinal Protozoa and Mange Parasites by a Floatation Technique. Journal of Pathology and Therapy, 36(pt. 4).

Telemann, W. (1908). Eine methode zur erleichterung der auffindung von parasiteneiern in den faeces. DMW-Deutsche Medizinische Wochenschrift, 34(35), 1510-1511.

Torgerson, P. R., Devleesschauwer, B., Praet, N., Speybroeck, N., Willingham, A. L., Kasuga, F., \& Gargouri, N. (2015). World Health Organization estimates of the global and regional disease burden of 11 foodborne parasitic diseases, 2010: a data synthesis. PLoS medicine, 12(12), e1001920.

Willis, H. H. (1921). A simple levitation method for the detection of hookworm ova. Medical Journal of Australia, 2(18).

Zonta, M. L., Oyhenart, E. E., \& Navone, G. T. (2010). Nutritional status, body composition, and intestinal parasitism among the Mbyá-Guaraní communities of Misiones, Argentina. American Journal of Human Biology: The Official Journal of the Human Biology Association, 22(2), 193200. 